\title{
COUNTING LATTICE POINTS IN CERTAIN RATIONAL POLYTOPES AND GENERALIZED DEDEKIND SUMS
}

\author{
KazUhito KozUKA
}

\begin{abstract}
Let $\mathcal{P} \subset \mathbf{R}^{n}$ be a rational convex polytope with vertices at the origin and on each positive coordinate axes. On the basis of the study on counting lattice points in $t \mathcal{P}$ with positive integer $t$, which is deeply connected with reciprocity laws for generalized Dedekind sums, we study the number of lattice points in the shifted polytope of $t \mathcal{P}$ by a fixed rational point. Certain generalized multiple Dedekind sums appear naturally in the main result.
\end{abstract}

Keywords: rational polytopes, lattice points, Ehrhart quasipolynomial, Dedekind sums.

\section{Introduction}

Let $\mathcal{P} \subset \mathbf{R}^{n}$ be a rational convex polytope and for $t \in \mathbf{N}$, put

$$
L_{\mathcal{P}}(t)=\sharp\left(t \mathcal{P} \cap \mathbf{Z}^{n}\right),
$$

the number of lattice points in $t \mathcal{P}$. It is known that $L_{\mathcal{P}}(t)$ is expressed as

$$
L_{\mathcal{P}}(t)=c_{n}(t) t^{n}+\cdots+c_{1}(t) t+c_{0}(t)
$$

with periodic functions $c_{0}(t), \cdots, c_{n}(t)$ and is called the Ehrhart quasipolynomial of $\mathcal{P}([15])$. Further the problem of finding an explicit expression of $L_{\mathcal{P}}(t)$ is deeply connected with reciprocity laws for certain generalized Dedekind sums. Historically, the first example appeared in [16], where Mordell studied the number of lattice points in the interior of the tetrahedron

$$
\mathcal{P}=\left\{(x, y, z) \in \mathbf{R}_{\geqslant 0}^{3} \mid \frac{x}{a}+\frac{y}{b}+\frac{z}{c} \leqslant 1\right\}
$$

for $a, b, c \in \mathbf{N}$ and obtained a formula connected with a three-term relation of the classical Dedekind sums.

2010 Mathematics Subject Classification: primary: 05A15, 11F20 
Generalizations for higher dimensional case are studied in [2], [3] and [4] etc., in which Dedekind-Rademacher sums or Fourier-Dedekind sums appear naturally. Here, along the content of this paper, put

$$
\mathcal{P}(\boldsymbol{a})=\left\{\left(x_{1}, \cdots, x_{n}\right) \in \mathbf{R}_{\geqslant 0}^{n} \mid a_{1} x_{1}+\cdots a_{n} x_{n} \leqslant 1\right\}
$$

and

$$
L(t: \boldsymbol{a})=L_{\mathcal{P}(\boldsymbol{a})}(t)
$$

for $\boldsymbol{a}=\left(a_{1}, \cdots, a_{n}\right) \in \mathbf{N}^{n}$. Let us recall the result for this case. It is obvious that $L(t: \boldsymbol{a})$ is equal to the Taylor coefficient of $z^{t}$ of the function

$$
\begin{aligned}
F(z: \boldsymbol{a}) & \stackrel{\text { def }}{=}\left(\prod_{i=1}^{n}\left(1+z^{a_{i}}+z^{2 a_{i}}+\cdots\right)\right)\left(1+z+z^{2}+\cdots\right) \\
& =\left(\prod_{i=1}^{n} \frac{1}{1-z^{a_{i}}}\right) \frac{1}{1-z} .
\end{aligned}
$$

Making use of this, Beck, Dias and Robins studied in [3] an explicit expression of $L(t: \boldsymbol{a})$ under the condition of $\operatorname{gcd}\left\{a_{i}, a_{j}\right\}=1$ for all $i \neq j$. In order to state the result precisely, let us define the Fourier-Dedekind sum by

$$
\sigma_{l}\left(c_{1}, \cdots, c_{n}: c\right)=\frac{1}{c} \sum_{\substack{\zeta^{c}=1 \\ \zeta \neq 1}} \frac{\zeta^{l}}{\left(\zeta^{c_{1}}-1\right) \cdots\left(\zeta^{c_{n}}-1\right)}
$$

for $c, c_{1}, \cdots, c_{n} \in \mathbf{N}$ and $l \in \mathbf{Z}$, and put

$$
R_{-t}(\boldsymbol{a})=-\operatorname{Res}\left(z^{-t-1} F(z: \boldsymbol{a}): z=1\right) .
$$

Then, it is shown in [3] that

$$
L(t: \boldsymbol{a})=R_{-t}(\boldsymbol{a})+(-1)^{n} \sum_{i=1}^{n} \sigma_{-t}\left(a_{1}, \cdots, \widehat{a_{i}}, \cdots, a_{n}, 1: a_{i}\right) .
$$

Note that if we put

$$
p(t: \boldsymbol{a})=\sharp\left\{\left(m_{1}, \cdots, m_{n}\right) \in \mathbf{Z}_{\geqslant 0}^{n} \mid a_{1} m_{1}+\cdots a_{n} m_{n}=t\right\},
$$

then

$$
L(t: \boldsymbol{a})=p(t:(\boldsymbol{a}, 1)),
$$

where $(\boldsymbol{a}, 1)=\left(a_{1}, \cdots, a_{n}, 1\right) \in \mathbf{N}^{n+1}$. In [4], Beck, Gessel and Komatsu studied a formula for the polynomial part of $p(t: \boldsymbol{a})$. From Theorem and Proposition of [4] and Remark 1 of [3], we see that $R_{-t}(\boldsymbol{a})$ equals the polynomial part of $p(t:(\boldsymbol{a}, 1))$ and is expressed as

$$
R_{-t}(\boldsymbol{a})=\frac{1}{a_{1} \cdots a_{n}} \sum_{m=0}^{n} \frac{(-1)^{m}}{(n-m) !} \sum_{\substack{p_{1}, \cdots, p_{n}, q \in \mathbf{Z}_{\geqslant 0} \\ p_{1}+\cdots+p_{n}+q=m}} a_{1}^{p_{1}} \cdots a_{n}^{p_{n}} \frac{B_{p_{1}} \cdots B_{p_{n}} B_{q}}{p_{1} ! \cdots p_{n} ! q !} t^{n-m}
$$

where $B_{p}$ is the $p$ th Bernoulli number. 
As for the value at $t=0$, it is known that if $\mathcal{P}$ is an integral polytope, $L_{\mathcal{P}}(t)$ is a polynomial of $t$ of which the constant term equals the Euler characteristic $\chi(\mathcal{P})$ of $\mathcal{P}$. It is also known that $\chi(\mathcal{P})=1$ if $\mathcal{P}$ is convex. In our case, since $a_{1} \cdots a_{n} \cdot \mathcal{P}(\boldsymbol{a})$ is integral and convex, we have $L(0, \boldsymbol{a})=1$. We note that this can also be interpreted as $L(0, \boldsymbol{a})=\sharp\left(0 \cdot \mathcal{P}(\boldsymbol{a}) \cap \mathbf{Z}^{n}\right)$. In addition the formula (1.2) also holds for $t=0$.

Now the classical Dedekind sum $s(a, b)$ is defined by

$$
s(a, b)=\sum_{\lambda \bmod b}\left(\left(\frac{\lambda}{b}\right)\right)\left(\left(\frac{a \lambda}{b}\right)\right),
$$

where $a \in \mathbf{Z}, b \in \mathbf{N}$ and

$$
((x))= \begin{cases}x-[x]-\frac{1}{2} & \text { if } x \notin \mathbf{Z} \\ 0 & \text { if } x \in \mathbf{Z} .\end{cases}
$$

If $a, b \in \mathbf{N}$ with $\operatorname{gcd}\{a, b\}=1$, we have a well-known reciprocity law such as

$$
s(a, b)+s(b, a)=\frac{1}{12}\left(\frac{b}{a}+\frac{a}{b}+\frac{1}{a b}\right)-\frac{1}{4} .
$$

([14], [18]). In the special case of $n=2$ and $t=0$, we have

$$
\sigma_{0}(a, 1: b)=-s(a, b)+\frac{1}{4}-\frac{1}{4 a b}
$$

and the formula (1.2), together with (1.3), naturally reduces to (1.5).

In this paper, as a generalization of $L(t: \boldsymbol{a})$, we study the formula for the number of the lattice points in the shifted polytope of $t \mathcal{P}(\boldsymbol{a})$ by a fixed rational point, namely the formula expressing $\sharp\left((-\boldsymbol{\alpha}+t \mathcal{P}(\boldsymbol{a})) \cap \mathbf{Z}^{n}\right)$ for $\boldsymbol{\alpha} \in \mathbf{Q}^{n}$. The special case of $n=2$, in which $\mathcal{P}(\boldsymbol{a})$ is a rectangled triangle in $\mathbf{R}^{2}$, is studied in [5]. In our main result, we enlarge the range of $t$ as $t \in \mathbf{Q}_{\geqslant 0}$ and multiple versions of the Dedekind-Rademacher sums will appear naturally. Let us give a description of each section.

In Section 2, we first recall the definition and basic properties of Bernoulli functions and give a definition of generalized Dedekind sums which appear in our main result.

In Section 3, as important tools for the study of lattice points in rational polytopes, we describe the integer-point transforms of rational polytopes or cones in $\mathbf{R}^{n}$ and well-known Brion's Theorem. Then we state the main result as a natural application of Brion's Theorem to the polytope $-\boldsymbol{\alpha}+t \mathcal{P}(\boldsymbol{a})$. As a Corollary of the main result, we also show a generalized reciprocity law for multiple DedekindRademacher sums.

In order to prove the main result, we prepare two equations as Lemmas in Section 4 and complete the proof in Section 5. 


\section{Notations and definitions}

Let $B_{p}(X)$ be the $p$ th Bernoulli polynomial defined by

$$
\frac{t e^{t X}}{e^{t}-1}=\sum_{p=0}^{\infty} B_{p}(X) \frac{t^{p}}{p !}
$$

and let $B_{p}=B_{p}(0)$, the $p$ th Bernoulli number. For any $x \in \mathbf{Q}$, write $x=[x]+\{x\}$ with $[x] \in \mathbf{Z}$ and $0 \leqslant\{x\}<1$ and define $\tilde{B}_{p}(x)=B_{p}(\{x\})$, which is periodic of period 1 and satisfies a distribution relation such as

$$
\sum_{\lambda \bmod k} \tilde{B}_{p}\left(x+\frac{\lambda}{k}\right)=k^{1-p} \tilde{B}_{p}(k x)
$$

for any $k \in \mathbf{N}$ and $x \in \mathbf{Q}$. Let $P=\left(p_{1}, \cdots, p_{n}\right) \in \mathbf{Z}_{\geqslant 0}^{n}, q \in \mathbf{Z}_{\geqslant 0}, \boldsymbol{a}=$ $\left(a_{1}, \cdots, a_{n}\right) \in \mathbf{Z}^{n}, b \in \mathbf{Z}, \boldsymbol{\alpha}=\left(\alpha_{1}, \cdots, \alpha_{n}\right) \in \mathbf{Q}^{n}$ and $\beta \in \mathbf{Q}$, and we define the following multiple Dedekind sum:

$$
\begin{aligned}
\mathcal{S}_{(P, q)} & \left(\begin{array}{ll}
\boldsymbol{a} & b \\
\boldsymbol{\alpha} & \beta
\end{array}\right) \\
\quad= & \sum_{\lambda_{1}, \cdots, \lambda_{n} \bmod b}\left(\prod_{j=1}^{n} \tilde{B}_{p_{j}}\left(\frac{\lambda_{j}+\alpha_{j}}{b}\right)\right) \tilde{B}_{q}\left(\frac{\sum_{j=1}^{n} a_{j}\left(\lambda_{j}+\alpha_{j}\right)}{b}+\beta\right) .
\end{aligned}
$$

In the special case of $n=1$, the sum is reduced to the classical Dedekind sum (1.4) as

In addition, we also have

$$
\mathcal{S}_{(1,1)}\left(\begin{array}{ll}
a & b \\
0 & 0
\end{array}\right)=\frac{1}{4}+s(a, b) .
$$

$$
\mathcal{S}_{(p, q)}\left(\begin{array}{cc}
a & b \\
\alpha & \beta
\end{array}\right)=\sum_{\lambda \bmod b} \tilde{B}_{p}\left(\frac{\lambda+\alpha}{b}\right) \tilde{B}_{q}\left(\frac{a(\lambda+\alpha)}{b}+\beta\right),
$$

which essentially includes the sums defined by Apostol as (1.3) in [1], by Rademacher as (1.3) in [17] and by Carlitz as (1.2) in [8], (1.7) in [10] and (1.12) in [12]. We also note that in [10] and [13], Carlitz had already studied the sum (2.2) in the case of $P=(1, \cdots, 1), \boldsymbol{\alpha}=(0, \cdots, 0)$ and $\beta=0$ with rather modified forms.

In the case of $P=(1, \cdots, 1), q=1, \boldsymbol{\alpha}=\mathbf{0}=(0, \cdots, 0)$ and $\beta=t / b$, the sum (2.2) is reduced to the Fourier-Dedekind sum (1.1) in such a way that

$$
\mathcal{S}_{(1, \cdots, 1,1)}\left(\begin{array}{cc}
\boldsymbol{a} & b \\
\mathbf{0} & t / b
\end{array}\right)=\sigma_{-t}(-\boldsymbol{a}, 1: b)+\frac{B_{1}^{n}}{b} .
$$

In the case of $(P, q)=\left(p_{1}, \cdots, p_{n}, q\right) \in \mathbf{Z}_{\geqslant 0}^{n+1}-\mathbf{N}^{n+1}$ and $\operatorname{gcd}\left(b, a_{j}\right)=1$ for $1 \leqslant j \leqslant n$, we can derive by $(2.1)$ that

$$
\mathcal{S}_{(P, q)}\left(\begin{array}{cc}
\boldsymbol{a} & b \\
\boldsymbol{\alpha} & \beta
\end{array}\right)=b^{n-\left(p_{1}+\cdots+p_{n}+q\right)}\left(\prod_{j=1}^{n} \tilde{B}_{p_{j}}\left(\alpha_{j}\right)\right) \tilde{B}_{q}(\boldsymbol{a} \cdot \boldsymbol{\alpha}+b \beta),
$$

where $\boldsymbol{a} \cdot \boldsymbol{\alpha}=a_{1} \alpha_{1}+\cdots+a_{n} \alpha_{n}$, the inner product of $\boldsymbol{a}$ and $\boldsymbol{\alpha}$. 


\section{Integer-point transforms}

Let $S \subset \mathbf{R}^{n}$ be a rational cone or polytope, The integer-point transform of $S$ is defined by

$$
\sigma\left(u_{1}, \cdots, u_{n}: S\right)=\sum_{\left(m_{1}, \cdots, m_{n}\right) \in S \cap \mathbf{Z}^{n}} u_{1}^{m_{1}} \cdots u_{n}^{m_{n}} .
$$

If $S$ is a polytope, the right-hand side of (3.1) is a finite sum. If $S$ is a cone, the right-hand side of (3.1) is a Laurent series of $u_{1}^{\varepsilon_{1}}, \cdots, u_{n}^{\varepsilon_{n}}$, where $\varepsilon_{j}=1$ or -1 for $1 \leqslant j \leqslant n$ and can also be expressed as a rational function of $u_{1}, \cdots, u_{n}$ (cf. Chapter 3.2 of $[6])$.

Let $\boldsymbol{a}=\left(a_{1}, \cdots, a_{n}\right) \in \mathbf{N}^{n}, b \in \mathbf{N}, \boldsymbol{\alpha}=\left(\alpha_{1}, \cdots, \alpha_{n}\right) \in \mathbf{Q}^{n}$ and $\beta \in \mathbf{Q}$. In what follows, we consider the range of $t$ as $t \in \mathbf{Q}_{\geqslant 0}$.

Proposition 3.1. Let $t \in \mathbf{Q}_{\geqslant 0}$ and let $K(t)$ denote the cone in $\mathbf{R}^{n+1}$ defined by

$$
\begin{aligned}
K(t)=\left\{\left(x_{1}, \cdots, x_{n}, y\right) \in \mathbf{R}^{n+1} \mid \sum_{j=1}^{n} a_{j}\left(x_{j}+\alpha_{j}\right)+b(y+\beta) \leqslant t\right. & \\
& \left.x_{j}+\alpha_{j} \geqslant 0(1 \leqslant j \leqslant n)\right\} .
\end{aligned}
$$

Then we have

$$
\begin{aligned}
\sigma\left(u_{1}, \cdots, u_{n}, v: K(t)\right) & \\
= & \sum_{0 \leqslant \lambda_{1}, \cdots, \lambda_{n} \leqslant b-1}\left(\prod_{j=1}^{n} \frac{u_{j}^{\lambda_{j}-\left[\alpha_{j}\right]}}{1-u_{j}^{b} v^{-a_{j}}}\right) \frac{v^{\left[-\frac{1}{b} \sum_{j=1}^{n} a_{j}\left(\lambda_{j}+\left\{\alpha_{j}\right\}\right)-\beta+\frac{t}{b}\right]}}{1-v^{-1}} \\
= & u_{1}^{-\alpha_{1}} \cdots u_{n}^{-\alpha_{n}} v^{-\beta+\frac{t}{b}} \sum_{0 \leqslant \lambda_{1}, \cdots, \lambda_{n} \leqslant b-1}\left(\prod_{j=1}^{n} \frac{\left(u_{j}^{b} v^{-a_{j}}\right)^{\frac{\lambda_{j}+\left\{\alpha_{j}\right\}}{b}}}{1-u_{j}^{b} v^{-a_{j}}}\right) \\
& \times \frac{v^{-\left\{-\frac{1}{b} \sum_{j=1}^{n} a_{j}\left(\lambda_{j}+\left\{\alpha_{j}\right\}\right)-\beta+\frac{t}{b}\right\}}}{1-v^{-1}} .
\end{aligned}
$$

Proof. If $\left(m_{1}, \cdots, m_{n}, m\right) \in K(t) \cap \mathbf{Z}^{n+1}$, then we have $m_{j}+\alpha_{j} \geqslant 0$ for each $1 \leqslant j \leqslant n$ and

$$
m \leqslant-\frac{1}{b} \sum_{j=1}^{n} a_{j}\left(m_{j}+\alpha_{j}\right)-\beta+\frac{t}{b} .
$$

This implies $m_{j}+\left[\alpha_{j}\right] \in \mathbf{Z}_{\geqslant 0}$ and we can express

$$
m_{j}=-\left[\alpha_{j}\right]+\lambda_{j}+b l_{j} \quad \text { with } 0 \leqslant \lambda_{j} \leqslant b-1 \text { and } l_{j} \in \mathbf{Z}_{\geqslant 0}
$$


and

$$
\begin{aligned}
m & =\left[-\frac{1}{b} \sum_{j=1}^{n} a_{j}\left(m_{j}+\alpha_{j}\right)-\beta+\frac{t}{b}\right]-l \quad \text { with } \quad l \in \mathbf{Z}_{\geqslant 0} \\
& =\left[-\frac{1}{b} \sum_{j=1}^{n} a_{j}\left(\lambda_{j}+\left\{\alpha_{j}\right\}\right)-\beta+\frac{t}{b}\right]-\sum_{j=1}^{n} a_{j} l_{j}-l .
\end{aligned}
$$

Hence

$$
\begin{aligned}
\sigma\left(u_{1}, \cdots, u_{n}, v: K(t)\right) & \sum_{l_{1}, \cdots, l_{n}, l \geqslant 0} \sum_{0 \leqslant \lambda_{1}, \cdots, \lambda_{n} \leqslant b-1}\left(\prod_{j=1}^{n} u_{j}^{-\left[\alpha_{j}\right]+\lambda_{j}}\right) v^{\left[-\frac{1}{b} \sum_{j=1}^{n} a_{j}\left(\lambda_{j}+\left\{\alpha_{j}\right\}\right)-\beta+\frac{t}{b}\right]} \\
& \times\left(\prod_{j=1}^{n}\left(u_{j}^{b} v^{-a_{j}}\right)^{l_{j}}\right) \cdot v^{-l} \\
= & \sum_{0 \leqslant \lambda_{1}, \cdots, \lambda_{n} \leqslant b-1}\left(\prod_{j=1}^{n} \frac{u_{j}^{\lambda_{j}-\left[\alpha_{j}\right]}}{1-u_{j}^{b} v^{-a_{j}}}\right) \frac{v^{\left[-\frac{1}{b} \sum_{j=1}^{n} a_{j}\left(\lambda_{j}+\left\{\alpha_{j}\right\}\right)-\beta+\frac{t}{b}\right]}}{1-v^{-1}} .
\end{aligned}
$$

Thus, we obtain (3.2) and equation (3.3) is directly derived from (3.2) by making use of $[x]=x-\{x\}$ for any $x \in \mathbf{Q}$.

Now suppose that $\boldsymbol{a}=\left(a_{1}, \cdots, a_{n}\right) \in \mathbf{N}^{n}$ with $\operatorname{gcd}\left\{a_{i}, a_{j}\right\}=1$ for all $i \neq j$ and as in the introduction, put

$$
\mathcal{P}(\boldsymbol{a})=\left\{\left(x_{1}, \cdots, x_{n}\right) \in \mathbf{R}_{\geqslant 0}^{n} \mid a_{1} x_{1}+\cdots+a_{n} x_{n} \leqslant 1\right\} .
$$

Let $\mathrm{A}_{1}, \mathrm{~A}_{2}, \cdots, \mathrm{A}_{n}$ denote the points $\left(\frac{1}{a_{1}}, 0, \cdots, 0\right),\left(0, \frac{1}{a_{2}}, 0, \cdots, 0\right), \cdots$, $\left(0, \cdots, 0, \frac{1}{a_{n}}\right)$, respectively. Then for $t>0$, the vertices of $t \mathcal{P}(\boldsymbol{a})$ are the origin and $t \mathrm{~A}_{1}, \cdots, t \mathrm{~A}_{n}$. For each $1 \leqslant i \leqslant n$, let $K_{i}(t)$ denote the tangent cone of $t \mathrm{~A}_{i}$. Then

$$
\begin{aligned}
K_{i}(t) & =\left\{\left(t-\mu_{i}\right) \overrightarrow{\mathrm{OA}_{i}}+\sum_{j \neq i} \mu_{j} \overrightarrow{\mathrm{A}_{i} \mathrm{~A}_{j}} \mid \mu_{1}, \cdots, \mu_{n} \geqslant 0\right\} \\
= & \left\{\left(x_{1}, \cdots, x_{n}\right) \in \mathbf{R}^{n} \mid a_{1} x_{1}+\cdots+a_{n} x_{n} \leqslant t,\right. \\
& \left.x_{j} \geqslant 0 \text { for } 1 \leqslant j \leqslant n \text { with } j \neq i\right\} .
\end{aligned}
$$

In addition, we put

$$
K_{0}(t)=\mathbf{R}_{\geqslant 0}^{n},
$$


which is the tangent cone of the origin for $t \mathcal{P}(\boldsymbol{a})$. Let $\boldsymbol{\alpha}=\left(\alpha_{1}, \cdots, \alpha_{n}\right) \in \mathbf{Q}^{n}$ and let us consider the shifted polytope

$$
\begin{array}{r}
-\boldsymbol{\alpha}+t \mathcal{P}(\boldsymbol{a})=\left\{\left(x_{1}, \cdots, x_{n}\right) \in \mathbf{R}^{n} \mid a_{1}\left(x_{1}+\alpha_{1}\right)+\cdots+a_{n}\left(x_{n}+\alpha_{n}\right) \leqslant t\right. \\
\left.x_{j}+\alpha_{i} \geqslant 0(1 \leqslant j \leqslant n)\right\}
\end{array}
$$

and put

$$
L(t: \boldsymbol{a}, \boldsymbol{\alpha})=\sharp\left((-\boldsymbol{\alpha}+t \mathcal{P}(\boldsymbol{a})) \cap \mathbf{Z}^{n}\right) .
$$

Then the vertices of $-\boldsymbol{\alpha}+t \mathcal{P}(\boldsymbol{a})$ are the points $-\boldsymbol{\alpha}$ and $-\boldsymbol{\alpha}+t \mathrm{~A}_{i}$ for $1 \leqslant i \leqslant n$ and their tangent cones are $-\boldsymbol{\alpha}+K_{0}(t)=-\boldsymbol{\alpha}+\mathbf{R}_{\geqslant 0}^{n}$ and

$$
\begin{array}{r}
-\boldsymbol{\alpha}+K_{i}(t)=\left\{\left(x_{1}, \cdots, x_{n}\right) \in \mathbf{R}^{n} \mid a_{1}\left(x_{1}+\alpha_{1}\right)+\cdots+a_{n}\left(x_{n}+\alpha_{n}\right) \leqslant t,\right. \\
\left.x_{j}+\alpha_{j} \geqslant 0 \text { for } 1 \leqslant j \leqslant n \text { with } j \neq i\right\},
\end{array}
$$

respectively. Applying (3.3), we see that

$$
\begin{aligned}
\sigma\left(u_{1}, \cdots,\right. & \left.u_{n},:-\boldsymbol{\alpha}+K_{i}(t)\right) \\
= & u_{1}^{-\alpha_{1}} \cdots u_{n}^{-\alpha_{n}} u_{i}^{\frac{t}{a_{i}}} \sum_{\substack{0 \leqslant \lambda_{1}, \cdots, \widehat{\lambda_{i}, \cdots, \lambda_{n} \leqslant a_{i}-1} \\
j \neq i}}\left(\prod_{j \neq u_{j}} \frac{\left(u_{j}^{a_{i}} u_{i}^{-a_{j}}\right)^{\frac{\lambda_{j}+\left\{\alpha_{j}\right\}}{a_{i}}}}{1-u_{j}^{a_{i}} u_{i}^{-a_{j}}}\right) \\
& \times \frac{u_{i}^{-\left\{-\frac{1}{a_{i}} \sum_{j \neq i} a_{j}\left(\lambda_{j}+\left\{\alpha_{j}\right\}\right)-\alpha_{i}+\frac{t}{a_{i}}\right\}}}{1-u_{i}^{-1}}
\end{aligned}
$$

for $1 \leqslant i \leqslant n$. For $i=0$, we have

$$
\begin{aligned}
\sigma\left(u_{1}, \cdots, u_{n},:-\boldsymbol{\alpha}+K_{0}(t)\right) & =\sum_{\left(m_{1}, \cdots, m_{n}\right) \in\left(-\boldsymbol{\alpha}+\mathbf{R}_{\geqslant 0}^{n}\right) \cap \mathbf{Z}^{n}} u_{1}^{m_{1}} \cdots u_{n}^{m_{n}} \\
& =\prod_{i=1}^{n} \sum_{m_{i} \geqslant-\left[\alpha_{i}\right]} u_{i}^{m_{i}}=\prod_{i=1}^{n} \frac{u_{i}^{-\left[\alpha_{i}\right]}}{1-u_{i}} \\
& =u_{1}^{-\alpha_{1}} \cdots u_{n}^{-\alpha_{n}} \prod_{i=1}^{n} \frac{u_{i}^{\left\{\alpha_{i}\right\}}}{1-u_{i}} .
\end{aligned}
$$

Now we have the following theorem due to Brion ([7] or Theorem 9.7 of [6]).

Theorem 3.2 (Brion). Suppose $\mathcal{P} \subset \mathbf{R}^{n}$ is a rational convex polytope. For each vertix $v$ of $\mathcal{P}$, let $K_{v}$ denote the tangent cone of $v$. Then we have

$$
\sigma\left(u_{1}, \cdots, u_{n}: \mathcal{P}\right)=\sum_{v: \text { a vertix of } \mathcal{P}} \sigma\left(u_{1}, \cdots, u_{n}: K_{v}\right)
$$


Applying Brion's Theorem to $\mathcal{P}=-\boldsymbol{\alpha}+t \mathcal{P}(\boldsymbol{a})$, we deduce that

$$
\sigma\left(u_{1}, \cdots, u_{n}:-\boldsymbol{\alpha}+t \mathcal{P}(\boldsymbol{a})\right)=\sum_{i=0}^{n} \sigma\left(u_{1}, \cdots, u_{n}:-\boldsymbol{\alpha}+K_{i}(t)\right) .
$$

For each $P=\left(p_{1}, \cdots, p_{n}\right) \in \mathbf{Z}_{\geqslant 0}^{n}$, we put $P_{i}=\left(p_{1}, \cdots, \widehat{p}_{i}, \cdots, p_{n}\right)$ for $1 \leqslant i \leqslant n$. Similarly we put $\boldsymbol{a}_{i}=\left(a_{1}, \cdots, \widehat{a_{i}}, \cdots, a_{n}\right)$ and $\boldsymbol{\alpha}_{i}=\left(\alpha_{1}, \cdots, \widehat{\alpha_{i}}, \cdots, \alpha_{n}\right)$. Then taking $u_{i}=e^{a_{i} x_{i}}$ for $1 \leqslant i \leqslant n$ and combining equations (3.7), (3.8), (3.9) and the definition (2.2), we obtain

$$
\begin{aligned}
e^{a_{1} \alpha_{1} x_{1}+\cdots+a_{n} \alpha_{n} x_{n}} & \sum_{\left(m_{1}, \cdots, m_{n}\right) \in(-\boldsymbol{\alpha}+t \mathcal{P}(\boldsymbol{a})) \cap \mathbf{Z}^{n}} e^{a_{1} m_{1} x_{1}+\cdots+a_{n} m_{n} x_{n}} \\
= & (-1)^{n} \sum_{i=1}^{n} e^{t x_{i}} \sum_{P=\left(p_{1}, \cdots, p_{n}\right) \in \mathbf{Z}_{\geqslant 0}^{n}} \mathcal{S}_{\left(P_{i}, p_{i}\right)}\left(\begin{array}{cc}
-\boldsymbol{a}_{i} & a_{i} \\
\boldsymbol{\alpha}_{i} & -\alpha_{i}+\frac{t}{a_{i}}
\end{array}\right) \\
& \times\left(\prod_{j \neq i} \frac{\left(a_{i} a_{j}\left(x_{j}-x_{i}\right)\right)^{p_{j}-1}}{p_{j} !}\right) \frac{\left(-a_{i} x_{i}\right)^{p_{i}-1}}{p_{i} !} \\
& +(-1)^{n} \sum_{P=\left(p_{1}, \cdots, p_{n}\right) \in \mathbf{Z}_{\geqslant 0}^{n}} \prod_{i=1}^{n} \frac{\tilde{B}_{p_{i}}\left(\alpha_{i}\right)}{p_{i} !}\left(a_{i} x_{i}\right)^{p_{i}-1}
\end{aligned}
$$

Here we give a supplementary explanation for the case of $t=0$. We define $K_{i}(0)$ by (3.4) or equivalently by (3.5) if $i \geqslant 1$ and by (3.6) if $i=0$. Then (3.7) and (3.8) are also valid for $t=0$. Further we have $-\boldsymbol{\alpha}+0 \cdot \mathcal{P}(\boldsymbol{a})=\{-\boldsymbol{\alpha}\}$ and we define $L(0: \boldsymbol{a}, \boldsymbol{\alpha})=\sharp\left(\{-\boldsymbol{\alpha}\} \cap \mathbf{Z}^{n}\right)$, which is 1 or 0 according as $\boldsymbol{\alpha} \in \mathbf{Z}^{n}$ or $\boldsymbol{\alpha} \notin \mathbf{Z}^{n}$. In the same way we can define $\sigma\left(u_{1}, \cdots, u_{n}:\{-\boldsymbol{\alpha}\}\right)=u^{-\alpha_{1}} \cdots u^{-\alpha_{n}}$ or 0 according as $\boldsymbol{\alpha} \in \mathbf{Z}^{n}$ or $\boldsymbol{\alpha} \notin \mathbf{Z}^{n}$. Since $\mathbf{Z}^{n}$ is discrete in $\mathbf{R}^{n}, L\left(t_{0}+\varepsilon: \boldsymbol{a}, \boldsymbol{\alpha}\right)$ and $\sigma\left(u_{1}, \cdots, u_{n}:-\boldsymbol{\alpha}+\left(t_{0}+\varepsilon\right) \mathcal{P}(\boldsymbol{a})\right)$ remain invariant for any fixed $t_{0} \in \mathbf{Q}_{\geqslant 0}$ and sufficiently small $\varepsilon \geqslant 0$. By considering the case of $t_{0}=0,(3.10)$ also holds for $t=0$.

Now for $t \in \mathbf{Q}_{\geqslant 0}$, we have

$$
L(t: \boldsymbol{a}, \boldsymbol{\alpha})=\sigma(1, \cdots, 1:-\boldsymbol{\alpha}+t \mathcal{P}(\boldsymbol{a})),
$$

which also equals the left-hand side of $(3.10)$ at $\left(x_{1}, \cdots, x_{n}\right)=(0, \cdots, 0)$. In the rest of this paper, we shall study the right-hand side of (3.10) and deduce the following main result.

Theorem 3.3. For any $t \in \mathbf{Q}_{\geqslant 0}$, we have

$$
L(t: \boldsymbol{a}, \boldsymbol{\alpha})=P(t: \boldsymbol{a}, \boldsymbol{\alpha})+(-1)^{n} \sum_{i=1}^{n} Q_{i}(t: \boldsymbol{a}, \boldsymbol{\alpha}),
$$


where

$$
\begin{aligned}
& P(t: \boldsymbol{a}, \boldsymbol{\alpha}) \\
= & \frac{1}{a_{1} \cdots a_{n}} \sum_{m=0}^{n} \sum_{\substack{m, \cdots, p_{n}, p \in \mathbf{Z}_{\geqslant 0} \\
p_{1}, \cdots+p_{n}+p=m}}(-1)^{m}\left(\prod_{i=1}^{n} \frac{a_{i}^{p_{i}} \tilde{B}_{p_{i}}\left(\alpha_{i}\right)}{p_{i} !}\right) \frac{\tilde{B}_{p}(-\boldsymbol{a} \cdot \boldsymbol{\alpha}+t)}{p !} \cdot \frac{t^{n-m}}{(n-m) !}
\end{aligned}
$$

symbolically

$$
=\frac{1}{a_{1} \cdots a_{n}}\left(t-\left(a_{1} \tilde{B}\left(\alpha_{1}\right)+\cdots+a_{n} \tilde{B}\left(\alpha_{n}\right)+\tilde{B}(-\boldsymbol{a} \cdot \boldsymbol{\alpha}+t)\right)\right)^{n} \frac{1}{n !}
$$

and

$$
Q_{i}(t: \boldsymbol{a}, \boldsymbol{\alpha})=\mathcal{S}_{(1, \cdots, 1)}\left(\begin{array}{cc}
-\boldsymbol{a}_{i} & a_{i} \\
\boldsymbol{\alpha}_{i} & -\alpha_{i}+\frac{t}{a_{i}}
\end{array}\right)-\frac{1}{a_{i}}\left(\prod_{j \neq i} \tilde{B}_{1}\left(\alpha_{j}\right)\right) \tilde{B}_{1}(-\boldsymbol{a} \cdot \boldsymbol{\alpha}+t) .
$$

Taking $t=0$ and making use of the symbolical expression as in (3.12), we can easily dedeuce a generalized reciprocity law for multiple Dedekind-Rademacher sums, which we show as the following.

Corollary 3.4. We have

$$
\begin{aligned}
a_{1} \cdots a_{n} \sum_{i=1}^{n} \mathcal{S}_{(1, \cdots, 1)}\left(\begin{array}{cc}
-\boldsymbol{a}_{i} & a_{i} \\
\boldsymbol{\alpha}_{i} & -\alpha_{i}
\end{array}\right) \\
=-\frac{1}{n !}\left(a_{1} \tilde{B}\left(\alpha_{1}\right)+\cdots+a_{n} \tilde{B}\left(\alpha_{n}\right)+\tilde{B}(-\boldsymbol{a} \cdot \boldsymbol{\alpha})\right)^{n} \\
\quad+\sum_{i=1}^{n}\left(\prod_{j \neq i} a_{j} \tilde{B}_{1}\left(\alpha_{j}\right)\right) \tilde{B}_{1}(-\boldsymbol{a} \cdot \boldsymbol{\alpha})+\varepsilon
\end{aligned}
$$

where $\varepsilon=(-1)^{n} a_{1} \cdots a_{n}$ or 0 according as $\boldsymbol{\alpha} \in \mathbf{Z}^{n}$ or $\boldsymbol{\alpha} \notin \mathbf{Z}^{n}$.

\section{Preliminary results}

Let $\boldsymbol{x}=\left(x_{1}, \cdots, x_{n}\right)$ and $\triangle(\boldsymbol{x})=\triangle\left(x_{1}, \cdots, x_{n}\right)=\prod_{1 \leqslant i<j \leqslant n}\left(x_{i}-x_{j}\right)$, the difference product of $x_{1}, \cdots, x_{n}$. Then as is well known for the Vandermonde determinant, we have

$$
\left|\begin{array}{ccccc}
x_{1}^{n-1} & x_{1}^{n-2} & \cdots & x_{1} & 1 \\
\vdots & \vdots & & \vdots & \vdots \\
x_{n}^{n-1} & x_{n}^{n-2} & \cdots & x_{n} & 1
\end{array}\right|=\triangle(\boldsymbol{x}) .
$$

For the proof of Theorem 3.3, we shall need the following two lemmas. 
Lemma 4.1. Let $n \geqslant 2$ and $N \in \mathbf{Z}_{\geqslant 0}$. Then we have

$$
\left|\begin{array}{ccccc}
x_{1}^{N} & x_{1}^{n-2} & \cdots & x_{1} & 1 \\
\vdots & \vdots & & \vdots & \vdots \\
x_{n}^{N} & x_{n}^{n-2} & \cdots & x_{n} & 1
\end{array}\right|=\triangle(\boldsymbol{x}) \sum_{\substack{p_{1}, \cdots, p_{n} \in \mathbf{Z}_{\geqslant 0} \\
p_{1}+\cdots+p_{n}=N-n+1}} x_{1}^{p_{1}} \cdots x_{n}^{p_{n}} .
$$

Lemma 4.2. Let $n, N \in \mathbf{Z}_{\geqslant 0}$ and $l \in \mathbf{Z}$. Then we have

$$
\sum_{j=0}^{N}(-1)^{j}\left(\begin{array}{c}
N \\
j
\end{array}\right)\left(\begin{array}{c}
l+j \\
n
\end{array}\right)= \begin{cases}(-1)^{N}\left(\begin{array}{c}
l \\
n-N
\end{array}\right) & \text { if } N \leqslant n \\
0 & \text { otherwise. }\end{cases}
$$

Concerning Lemma 4.1, let us recall the Schur polynomial, which is defined by

$$
s\left(\boldsymbol{x}:\left(\lambda_{j}\right)\right)=\frac{\operatorname{det}\left(x_{i}^{\lambda_{j}+n-j}\right)}{\triangle(\boldsymbol{x})}
$$

for $\left(\lambda_{j}\right)=\left(\lambda_{1}, \cdots, \lambda_{n}\right) \in \mathbf{Z}_{\geqslant 0}$ with $\lambda_{1} \geqslant \cdots \geqslant \lambda_{n}$ and expressed by making use of the corresponding Young diagrams for $\left(\lambda_{j}\right)$. If $N \geqslant n-1,(4.1)$ is a direct consequence of the special case of $\left(\lambda_{j}\right)=(N-n+1,0, \cdots, 0)$, in which the Schur polynomial becomes the complete symmetric polynomial of degree $N-n+1$ in $n$ variables $x_{1}, \cdots, x_{n}$. Direct proof for this case is also possible by making use of induction on $N$. Note that in the case of $0 \leqslant N<n-1$, (4.1) is also valid since both sides become 0 .

As for Lemma 4.2, consider the following equation

$$
T^{l}(1+T)^{N}=\sum_{j=0}^{N}\left(\begin{array}{c}
N \\
j
\end{array}\right) T^{l+j} .
$$

Differentiating both sides $n$ times, we obtain

$$
\sum_{j=0}^{N}\left(\begin{array}{c}
n \\
j
\end{array}\right) \frac{d^{n-j} T^{l}}{d T^{n-j}} \cdot \frac{d^{j}}{d T^{j}}(1+T)^{N}=\sum_{j=0}^{N}\left(\begin{array}{c}
N \\
j
\end{array}\right) \frac{d^{n} T^{l+j}}{d T^{n}},
$$

namely

$$
\begin{aligned}
\sum_{j=0}^{N}\left(\begin{array}{c}
n \\
j
\end{array}\right)\left(\begin{array}{c}
l \\
n-j
\end{array}\right)(n-j) ! T^{l-n+j} \cdot\left(\begin{array}{c}
N \\
j
\end{array}\right) j !(1+T)^{N-j} & \\
& =\sum_{j=0}^{N}\left(\begin{array}{c}
N \\
j
\end{array}\right)\left(\begin{array}{c}
l+j \\
n
\end{array}\right) n ! T^{l+j-n} .
\end{aligned}
$$

By taking $T=-1$, the result follows immediately. 


\section{Proof of Theorem 3.3}

In order to study the right-hand side of (3.10), we put $Z_{0}=\mathbf{Z}_{\geqslant 0}^{n}$ and $Z_{1}=$ $\mathbf{N}^{n}$, and introduce the following functions $G_{k}(\boldsymbol{x})=G_{k}\left(x_{1}, \cdots, x_{n}\right)$ and $H_{k}(\boldsymbol{x})=$ $H_{k}\left(x_{1}, \cdots, x_{n}\right)$ for $k=0,1$ :

$$
\begin{aligned}
G_{k}(\boldsymbol{x})= & \sum_{i=1}^{n} e^{t x_{i}} \sum_{P=\left(p_{1}, \cdots, p_{n}\right) \in Z_{k}} \mathcal{S}_{\left(P_{i}, p_{i}\right)}\left(\begin{array}{cc}
-\boldsymbol{a}_{i} & a_{i} \\
\boldsymbol{\alpha}_{i} & -\alpha_{i}+\frac{t}{a_{i}}
\end{array}\right) \\
& \times\left(\prod_{j \neq i} \frac{\left(a_{i} a_{j}\left(x_{j}-x_{i}\right)\right)^{p_{j}-1}}{p_{j} !}\right) \frac{\left(-a_{i} x_{i}\right)^{p_{i}-1}}{p_{i} !} \\
H_{k}(\boldsymbol{x})= & \frac{1}{a_{1} \cdots a_{n}} \sum_{i=1}^{n} e^{t x_{i}} \sum_{P=\left(p_{1}, \cdots, p_{n}\right) \in Z_{k}}\left(\prod_{j \neq i} a_{j}^{p_{j}} \tilde{B}_{p_{j}}\left(\alpha_{j}\right)\right) \tilde{B}_{p_{i}}(-\boldsymbol{a} \cdot \boldsymbol{\alpha}+t) \\
& \times\left(\prod_{j \neq i} \frac{\left(x_{j}-x_{i}\right)^{p_{j}-1}}{p_{j} !}\right) \frac{\left(-x_{i}\right)^{p_{i}-1}}{p_{i} !} .
\end{aligned}
$$

Then by (2.4), we see that

$$
G_{0}(\boldsymbol{x})-G_{1}(\boldsymbol{x})=H_{0}(\boldsymbol{x})-H_{1}(\boldsymbol{x}) .
$$

Taking $\boldsymbol{x}=(x, \cdots, x)$, we have

$$
G_{1}(x, \cdots, x)=e^{t x} \sum_{i=1}^{n} \sum_{p=1}^{\infty} \mathcal{S}_{(1, \cdots, 1, p)}\left(\begin{array}{cc}
-\boldsymbol{a}_{i} & a_{i} \\
\boldsymbol{\alpha}_{i} & -\alpha_{i}+\frac{t}{a_{i}}
\end{array}\right) \frac{\left(-a_{i} x\right)^{p-1}}{p !}
$$

and

$$
H_{1}(x, \cdots, x)=e^{t x} \sum_{i=1}^{n} \frac{1}{a_{i}} \sum_{p=1}^{\infty}\left(\prod_{j \neq i} \tilde{B}_{1}\left(\alpha_{j}\right)\right) \tilde{B}_{p}(-\boldsymbol{a} \cdot \boldsymbol{\alpha}+t) \frac{(-x)^{p-1}}{p !} .
$$

Especially for $\boldsymbol{x}=(0, \cdots, 0)$, we have

$$
G_{1}(0, \cdots, 0)=\sum_{i=1}^{n} \mathcal{S}_{(1, \cdots, 1,1)}\left(\begin{array}{cc}
-\boldsymbol{a}_{i} & a_{i} \\
\boldsymbol{\alpha}_{i} & -\alpha_{i}+\frac{t}{a_{i}}
\end{array}\right)
$$

and

$$
H_{1}(0, \cdots, 0)=\sum_{i=1}^{n} \frac{1}{a_{i}}\left(\prod_{j \neq i} \tilde{B}_{1}\left(\alpha_{j}\right)\right) \tilde{B}_{1}(-\boldsymbol{a} \cdot \boldsymbol{\alpha}+t) .
$$

As for $H_{0}(\boldsymbol{x})$, we first note that

$$
\left.x_{i}^{p_{i}-1}\left(\prod_{j \neq i}\left(x_{j}-X\right)^{p_{j}}\right)\left(x_{i}-X\right)^{p}\right|_{X=x_{i}}= \begin{cases}x_{i}^{p_{i}-1} \prod_{j \neq i}\left(x_{j}-x_{i}\right)^{p_{j}} & \text { if } \quad p=0 \\ 0 & \text { if } \quad p \geqslant 1 .\end{cases}
$$


for $1 \leqslant i \leqslant n$. Changing the roles of $p_{i}$ and $p$, we can express

$$
\begin{aligned}
H_{0}(\boldsymbol{x})= & \frac{1}{a_{1} \cdots a_{n}} \sum_{i=1}^{n} e^{t x_{i}} \sum_{P=\left(p_{1}, \cdots, p_{n}\right) \in \mathbf{Z}_{\geqslant 0}^{n}} \sum_{p=0}^{\infty}\left(\prod_{j=1}^{n} \frac{a_{j}^{p_{j}} \tilde{B}_{p_{j}}\left(\alpha_{j}\right)}{p_{j} !}\right) \frac{\tilde{B}_{p}(-\boldsymbol{a} \cdot \boldsymbol{\alpha}+t)}{p !} \\
& \times \frac{\left.\left(-x_{i}\right)^{p-1} \prod_{j=1}^{n}\left(x_{j}-X\right)^{p_{j}}\right|_{X=x_{i}}}{\prod_{j \neq i}\left(x_{j}-x_{i}\right)} .
\end{aligned}
$$

For each $P=\left(p_{1}, \cdots, p_{n}\right) \in \mathbf{Z}_{\geqslant 0}^{n}$ and $p \in \mathbf{Z}_{\geqslant 0}$, we put

$$
\mathcal{B}(P, p)=\left(\prod_{j=1}^{n} \frac{a_{j}^{p_{j}} \tilde{B}_{p_{j}}\left(\alpha_{j}\right)}{p_{j} !}\right) \frac{\tilde{B}_{p}(-\boldsymbol{a} \cdot \boldsymbol{\alpha}+t)}{p !}
$$

and

$$
s(P)=p_{1}+\cdots+p_{n},
$$

and express

$$
\prod_{j=1}^{n}\left(x_{i}-X\right)^{p_{j}}=\sum_{k=0}^{s(P)} c_{k}(\boldsymbol{x}: P) X^{k}
$$

with $c_{k}(\boldsymbol{x}: P) \in \mathbf{Z}\left[x_{1}, \cdots, x_{n}\right]$. Then

$$
\begin{aligned}
& \triangle(\boldsymbol{x}) H_{0}(\boldsymbol{x}) \\
& =\frac{1}{a_{1} \cdots a_{n}} \sum_{p=0}^{\infty} \sum_{P \in \mathbf{Z}_{\geqslant 0}^{n}} \mathcal{B}(P, p) \sum_{i=1}^{n} e^{t x_{i}} \triangle\left(x_{1}, \cdots, \widehat{x_{i}}, \cdots, x_{n}\right)(-1)^{p+n-i-1} x_{i}^{p-1} \\
& \times \sum_{k=0}^{s(P)} c_{k}(\boldsymbol{x}: P) x_{i}^{k} \\
& =\frac{(-1)^{n}}{a_{1} \cdots a_{n}} \sum_{p=0}^{\infty} \sum_{P \in \mathbf{Z}_{\geqslant 0}^{n}}(-1)^{p} \mathcal{B}(P, p) \sum_{k=0}^{s(P)} c_{k}(\boldsymbol{x}: P)\left|\begin{array}{ccccc}
e^{t x_{1}} x_{1}^{p+k-1} & x_{1}^{n-2} & \cdots & x_{1} & 1 \\
\vdots & \vdots & & \vdots & \vdots \\
e^{t x_{n}} x_{n}^{p+k-1} & x_{n}^{n-2} & \cdots & x_{n} & 1
\end{array}\right| \\
& =\frac{(-1)^{n}}{a_{1} \cdots a_{n}} \sum_{p=0}^{\infty} \sum_{P \in \mathbf{Z}_{\geqslant 0}^{n}}(-1)^{p} \mathcal{B}(P, p) \sum_{k=0}^{s(P)} c_{k}(\boldsymbol{x}: P) \\
& \times \sum_{m=0}^{\infty} \frac{t^{m}}{m !}\left|\begin{array}{ccccc}
x_{1}^{m+p+k-1} & x_{1}^{n-2} & \cdots & x_{1} & 1 \\
\vdots & \vdots & & \vdots & \vdots \\
x_{n}^{m+p+k-1} & x_{n}^{n-2} & \cdots & x_{n} & 1
\end{array}\right| .
\end{aligned}
$$


Applying Lemma 4.1, we have

$$
\left|\begin{array}{ccccc}
x_{1}^{m+p+k-1} & x_{1}^{n-2} & \cdots & x_{1} & 1 \\
\vdots & \vdots & & \vdots & \vdots \\
x_{n}^{m+p+k-1} & x_{n}^{n-2} & \cdots & x_{n} & 1
\end{array}\right|=\triangle(\boldsymbol{x}) \sum_{\substack{Q=\left(q_{1}, \cdots, q_{n}\right) \in \mathbf{Z}_{\geqslant 0}^{n} \\
s(Q)=m+p+k-n}} x_{1}^{q_{1}} \cdots x_{n}^{q_{n}}
$$

except for the case of $m=p=k=0$. If $m=p=k=0$, the determinant above becomes

$$
\left|\begin{array}{ccccc}
x_{1}^{-1} & x_{1}^{n-2} & \cdots & x_{1} & 1 \\
\vdots & \vdots & & \vdots & \vdots \\
x_{n}^{-1} & x_{n}^{n-2} & \cdots & x_{n} & 1
\end{array}\right|=\frac{(-1)^{n-1}}{x_{1} \cdots x_{n}} \triangle(\boldsymbol{x}) .
$$

Hence we deduce that

$$
\begin{aligned}
& H_{0}(\boldsymbol{x}) \\
& =\frac{(-1)^{n}}{a_{1} \cdots a_{n}} \sum_{p=0}^{\infty} \sum_{P \in \mathbf{Z}_{\geqslant 0}^{n}}(-1)^{p} \mathcal{B}(P, p) \sum_{k=0}^{s(P)} c_{k}(\boldsymbol{x}: P) \sum_{m=0}^{\infty} \frac{t^{m}}{m !} \sum_{\substack{Q=\left(q_{1}, \cdots, q_{n}\right) \in \mathbf{Z}_{\geqslant 0}^{n} \\
s(Q)=m+p+k-n}} x_{1}^{q_{1}} \cdots x_{n}^{q_{n}} \\
& \quad-\frac{1}{a_{1} \cdots a_{n}} \sum_{P \in \mathbf{Z}_{\geqslant 0}^{n}} \frac{\mathcal{B}(P, 0) c_{0}(\boldsymbol{x}: P)}{x_{1} \cdots x_{n}} .
\end{aligned}
$$

Now taking $\boldsymbol{x}=(x, \cdots, x)$, we have

$$
\prod_{j=1}^{n}\left(x_{j}-X\right)^{p_{j}}=(x-X)^{s(P)}=\sum_{k=0}^{s(P)}\left(\begin{array}{c}
s(P) \\
k
\end{array}\right) x^{s(P)-k}(-1)^{k} X^{k}
$$

which implies

$$
c_{k}(x, \cdots, x: P)=\left(\begin{array}{c}
s(P) \\
k
\end{array}\right)(-1)^{k} x^{s(P)-k} .
$$

Hence

$$
\begin{aligned}
H_{0}(x, \cdots, x)= & \frac{(-1)^{n}}{a_{1} \cdots a_{n}} \sum_{p=0}^{\infty} \sum_{P \in \mathbf{Z}_{\geqslant 0}^{n}} \mathcal{B}(P, p) \sum_{k=0}^{s(P)}\left(\begin{array}{c}
s(P) \\
k
\end{array}\right)(-1)^{p+k} \\
& \times \sum_{m=0}^{\infty} \frac{t^{m}}{m !} \sum_{\substack{Q \in \mathbf{Z}_{\geqslant 0}^{n} \\
s(Q)=m+p+k-n}} x^{s(P)+s(Q)-k} \\
& -\frac{1}{a_{1} \cdots a_{n}} \sum_{P \in \mathbf{Z}_{\geqslant 0}^{n}} \mathcal{B}(P, 0) x^{s(P)-n} .
\end{aligned}
$$


Note that for any $l \in \mathbf{Z}_{\geqslant 0}$, the number of $Q \in \mathbf{Z}_{\geqslant 0}^{n}$ satisfying $s(Q)=l$ is what is called the number of repeated combination and equals $\left(\begin{array}{c}l+n-1 \\ n-1\end{array}\right)$. It follows that

$$
\begin{aligned}
H_{0}(x, \cdots, x)= & \frac{(-1)^{n}}{a_{1} \cdots a_{n}} \sum_{p=0}^{\infty} \sum_{P \in \mathbf{Z}_{\geqslant 0}^{n}} \mathcal{B}(P, p) \sum_{k=0}^{s(P)}\left(\begin{array}{c}
s(P) \\
k
\end{array}\right)(-1)^{p+k} \\
& \times \sum_{m=0}^{\infty} \frac{t^{m}}{m !}\left(\begin{array}{c}
m+p+k-1 \\
n-1
\end{array}\right) x^{m+p+s(P)-n} \\
& -\frac{(-1)^{n}}{a_{1} \cdots a_{n}} \sum_{P \in \mathbf{Z}_{\geqslant 0}^{n}} \mathcal{B}(P, 0)\left(\begin{array}{c}
-1 \\
n-1
\end{array}\right) x^{s(P)-n} \\
& -\frac{1}{a_{1} \cdots a_{n}} \sum_{P \in \mathbf{Z}_{\geqslant 0}^{n}} \mathcal{B}(P, 0) x^{s(P)-n} .
\end{aligned}
$$

Note that the last two summations in the right-hand side of this equation are canceled since $\left(\begin{array}{c}-1 \\ n-1\end{array}\right)=(-1)^{n-1}$. Then applying Lemma 4.2 , we see that

$$
\begin{aligned}
& H_{0}(x, \cdots, x) \\
= & \frac{(-1)^{n}}{a_{1} \cdots a_{n}} \sum_{p=0}^{\infty} \sum_{\substack{P \in \mathbf{Z}_{\geqslant 0}^{n} \\
s(P) \leqslant n-1}} \mathcal{B}(P, p) \sum_{m=0}^{\infty} \frac{t^{m}}{m !}(-1)^{p+s(P)}\left(\begin{array}{c}
m+p-1 \\
n-1-s(P)
\end{array}\right) x^{m+p+s(P)-n} .
\end{aligned}
$$

Now we see from (3.10) that

$$
\begin{aligned}
& L(t: \boldsymbol{a}, \boldsymbol{\alpha})=\text { constant term of } \\
& \qquad(-1)^{n}\left(G_{0}(x, \cdots, x)+\sum_{P=\left(p_{1}, \cdots, p_{n}\right) \in \mathbf{Z}_{\geqslant 0}^{n}}\left(\prod_{i=1}^{n} \frac{a_{i}^{p_{i}-1} \tilde{B}_{p_{i}}\left(\alpha_{i}\right)}{p_{i} !}\right) x^{s(P)-n}\right) .
\end{aligned}
$$

From (5.1), (5.2) and (5.3), we also see that the constant term of $G_{0}(x, \cdots, x)-H_{0}(x, \cdots, x)$ equals

$$
\begin{aligned}
& G_{1}(0, \cdots, 0)-H_{1}(0, \cdots, 0) \\
& \quad=\sum_{i=1}^{n}\left(\mathcal{S}_{(1, \cdots, 1,1)}\left(\begin{array}{cc}
-\boldsymbol{a}_{i} & a_{i} \\
\boldsymbol{\alpha}_{i} & -\alpha_{i}+\frac{t}{a_{i}}
\end{array}\right)-\frac{1}{a_{i}}\left(\prod_{j \neq i} \tilde{B}_{1}\left(\alpha_{j}\right)\right) \tilde{B}_{1}(-\boldsymbol{a} \cdot \boldsymbol{\alpha}+t)\right) .
\end{aligned}
$$


It follows from (5.4) that

$$
\begin{aligned}
& L(t: \boldsymbol{a}, \boldsymbol{\alpha}) \\
& =(-1)^{n} \sum_{i=1}^{n}\left(\mathcal{S}_{(1, \cdots, 1)}\left(\begin{array}{cc}
-\boldsymbol{a}_{i} & a_{i} \\
\boldsymbol{\alpha}_{i} & -\alpha_{i}+\frac{t}{a_{i}}
\end{array}\right)-\frac{1}{a_{i}}\left(\prod_{j \neq i} \tilde{B}_{1}\left(\alpha_{j}\right)\right) \tilde{B}_{1}(-\boldsymbol{a} \cdot \boldsymbol{\alpha}+t)\right) \\
& +\frac{1}{a_{1} \cdots a_{n}} \sum_{\substack{P \in \mathbf{Z}_{\geqslant 0}^{n} \\
s(P) \leqslant n-1}} \sum_{\substack{p, m \geqslant 0 \\
s(P)+m+p=n}}(-1)^{p+s(P)} \mathcal{B}(P, p) \frac{t^{m}}{m !} \\
& +\frac{(-1)^{n}}{a_{1} \cdots a_{n}} \sum_{\substack{P=\left(p_{1}, \cdots, p_{n}\right) \in \mathbf{Z}_{\geqslant 0}^{n} \\
s(P)=n}} \prod_{i=1}^{n} \frac{a_{i}^{p_{i}} \tilde{B}_{p_{i}}\left(\alpha_{i}\right)}{p_{i} !} \\
& =\frac{1}{a_{1} \cdots a_{n}} \sum_{\substack{P=\left(p_{1}, \cdots, p_{n}\right) \in \mathbf{Z}_{\geqslant 0}^{n} \\
s(P) \leqslant n}} \sum_{\substack{p, m \geqslant 0 \\
s(P)+m+p=n}}(-1)^{s(P)+p} \mathcal{B}(P, p) \frac{t^{m}}{m !} \\
& +(-1)^{n} \sum_{i=1}^{n}\left(\mathcal{S}_{(1, \cdots, 1)}\left(\begin{array}{cc}
-\boldsymbol{a}_{i} & a_{i} \\
\boldsymbol{\alpha}_{i} & -\alpha_{i}+\frac{t}{a_{i}}
\end{array}\right)-\frac{1}{a_{i}}\left(\prod_{j \neq i} \tilde{B}_{1}\left(\alpha_{j}\right)\right) \tilde{B}_{1}(-\boldsymbol{a} \cdot \boldsymbol{\alpha}+t)\right)
\end{aligned}
$$

which is easily transformed into the right-hand side of (3.11). This completes the proof of Theorem 3.3.

As for relations to preceding results mainly by Beck, Carlitz and Rademacher, we note the following.

Remark 5.1. In the case of $\boldsymbol{\alpha}=(0, \cdots, 0)$ and $t \in \mathbf{Z}_{\geqslant 0}, P(t: \boldsymbol{a}, \boldsymbol{\alpha})$ reduces to the right-hand side of $(1.3)$ and $Q_{i}(t: \boldsymbol{a}, \boldsymbol{\alpha})$ to $\sigma_{-t}\left(a_{1}, \cdots, \widehat{a_{i}}, \cdots, a_{n}: a_{i}\right)$ by virtue of (2.3). Hence (3.11) reduces to the formula (1.2).

Remark 5.2. In the case of $n=2$ and $t=0$, some calculations show that (3.13) reduces to the reciprocity law for Dedekind-Rademacher sums (Theorem 2 of [17] or the formula in the case $p=1$ for (4.4) of [11]). In addition, multiplying both sides of (3.10) by $\left(x_{1}-x_{2}\right) x_{1} x_{2}$ and examining the coefficient of $x_{1}^{r} x_{2}^{s}$ carefully for each $r, s \in \mathbf{Z}_{\geqslant 0}$, we can also derive the formula (2.15) of [12], which also reduces to (3.2) of [8] and (4.1) of [9] if $\boldsymbol{\alpha} \in \mathbf{Z}^{2}$.

Acknowledgements. The author would like to express his deep gratitude to Professor Beck for giving him some encouraging suggestions.

\section{References}

[1] T.M. Apostol, Generalized Dedekind sums and transformation formulae of certain Lambert series, Duke Math. J. 17 (1950), 147-157.

[2] M. Beck, Counting lattice points by means of the residue theorem, Ramanujan J. 4 (2000), 299-310. 
[3] M. Beck, R. Diaz and S. Robins, The Frobenius problem, rational polytopes, and Fourier-Dedekind sums, J. Number Theory 96 (2002), 1-21.

[4] M. Beck, I.M. Gessel and T. Komatsu, The polynomial part of a restricted partition function related to the Frobenius problem, Electronic J. Combin. 8, no.1 (2001), N7.

[5] M. Beck and S. Robins, Explicit and efficient formulas for the lattice point count in rational polygons using Dedekind-Rademacher sums, Discrete and Comp. Geom. 27 (2002), 443-459.

[6] M. Beck and S. Robins, Computing the continuous discretely: Integerpoint enumeration in polyhedra, Undergraduate Texs in Mathematics, Springer, New York, 2007.

[7] M. Brion, Points entiers dans les polyèdres convexes, Ann. Sci. École Norm. Sup (4) 21, no. 4 (1988), 653-663.

[8] L. Carlitz, Some theorems on generalized Dedekind sums, Pacific J. Math. 3, no.3 (1953), 513-522.

[9] L. Carlitz, Dedekind sums and Lambert series, Proc. Amer. Math. Soc. 5 (1954), 580-584.

[10] L. Carlitz, A note on generalized Dedekind sums, Duke. Math. J. 21 (1954), 399-403.

[11] L. Carlitz, Generalized Dedekind sums, Math. Zeit. 85 (1964), 83-90.

[12] L. Carlitz, Some theorems on generalized Dedekind-Rademacher sums, Pacific J. Math. 75 (1975), 347-358.

[13] L. Carlitz, Many-term relations for multiple Dedekind sums, Indian J. Math. 20 (1978), 77-89.

[14] R. Dedekind, Erläuterungen zu zwei Fragmenten von Riemann, Gesammelte mathematische Werke, Bd. I. S, 159-173.

[15] E. Ehrhart, Sur un problème de géométrie diophantienne linéaire II, J. reine angew. Math. 227 (1967), 25-49.

[16] L. Mordell, Lattice points in a tetrahedron and generalized Dedekind sums, J. Indian Math. Soc. (N.S) 15 (1951), 41-46.

[17] H. Rademacher, Some remarks on certain generalized Dedekind sums, Acta. Arith. 9 (1964), 97-105.

[18] H. Rademacher and A. Whiteman, Theorems on Dedekind sums, Amer. J. Math. 63 (1941), 377-407.

Address: Kazuhito Kozuka: Department of Mathematics, National Institute of Technology, Miyakonojo College, Miyakonojo, Miyazaki 885-8567, Japan.

E-mail: k31k@cc.miyakonojo-nct.ac.jp

Received: 28 December 2015; revised: 2 June 2016 